\title{
Non-repetitive Tilings
}

\author{
James D. Currie* \\ Department of Mathematics and Statistics \\ University of Winnipeg \\ Winnipeg, Manitoba \\ Canada R3B 2E9 \\ Fax: (204)-786-1824 \\ E-mail: currie@uwpg02.uwinnipeg.ca \\ Jamie Simpson \\ School of Mathematics and Statistics \\ Curtin University of Technology \\ Perth, Western Australia 6845 \\ E-mail: simpson@cs.curtin.edu.au
}

Submitted: November 9, 2001; Accepted: July 3, 2002.

MR Subject Classifications: 05B45, 05B30, 11B99

\begin{abstract}
In 1906 Axel Thue showed how to construct an infinite non-repetitive (or squarefree) word on an alphabet of size 3. Since then this result has been rediscovered many times and extended in many ways. We present a two-dimensional version of this result. We show how to construct a rectangular tiling of the plane using 5 symbols which has the property that lines of tiles which are horizontal, vertical or have slope +1 or -1 contain no repetitions. As part of the construction we introduce a new type of word, one that is non-repetitive up to $\bmod k$, which is of interest in itself. We also indicate how our results might be extended to higher dimensions.
\end{abstract}

\section{Introduction}

The word 'barbarian' can be written as $\mathbf{y y z}$ where $\mathbf{y}=$ 'bar' and $\mathbf{z}=$ 'ian'. Since the block y repeats next to itself in 'barbarian', we say that 'barbarian' is repetitive. Conversely,

*This work was supported by an NSERC operating grant. 
a word such as 'civilized', in which no two adjacent blocks are identical, is called nonrepetitive; thus, a word $\mathbf{w}$ is non-repetitive if one cannot write $\mathbf{w}=\mathbf{x y y z}$ with $\mathbf{y}$ a non-empty word. A set of letters $\Sigma$ is an alphabet, and the set of finite words over $\Sigma$ is denoted by $\Sigma^{*}$. We will use boldface letters to represent words and ordinary lower case letters for the letters which make up the word.

The study of non-repetitive words is an area of combinatorics on words reaching back to at least the beginning of the twentieth century. Thue [6] proved in 1906 that there are arbitrarily long non-repetitive words on 3 symbols. Infinite non-repetitive words ${ }^{1}$ have been used to build counter-examples in such diverse areas as algebra and dynamical systems $[2,3,5,4]$. Combinatorics on words can also be viewed as theoretical crystallography, where the tilings are one-dimensional. In algebra, sequences of symbols are basic objects. To study dynamical systems or crystals, it makes sense to consider higher dimensional analogs of sequences, i.e. arrays or tilings.

Non-repetitive tilings were briefly examined in [1]. The authors Bean, Ehrenfeucht and McNulty claim that there one can label each lattice point of the plane using the symbols $\{a, b, c\}$ such that no two adjacent rectangles receive the same labelling. Although they don't say so explicitly, it is clear that the rectangles considered are those with horizontal and vertical sides, that is, sets of lattice points of the form $\left\{(i, j): i_{0} \leq i \leq i_{1}, j_{0} \leq j \leq j_{1}\right\}$. Also, adjacencies are either vertical or horizontal (not diagonal). Their theorem is thus easily shown to be equivalent to the following:

Lemma 1.1 There is a labelling $f: \mathbb{Z}^{2} \rightarrow\{1,2,3\}$ of the lattice points of the plane, such that the horizontal bisequences $\left\{f\left(i, j_{0}\right)\right\}_{i=-\infty}^{\infty}$, and the vertical bisequences $\left\{f\left(i_{0}, j\right)\right\}_{j=-\infty}^{\infty}$ are non-repetitive for each $i_{0}, j_{0} \in \mathbb{Z}$.

For a proof, the authors of [1] give a map which replaces symbols by $13 \times 13$ arrays of symbols. Iterating this map they build an assignment of symbols to lattice points of the plane. Unfortunately, what is undoubtedly a typographical error in their paper gives the symbol $b$ next to itself in the last two positions of the ninth row of the image of $a$. We give here a new and much simpler proof of Lemma 1.1.

Proof of Lemma 1.1: Let $\mathbf{w}=\left\{c_{k}\right\}_{k=-\infty}^{\infty}$ be a non-repetitive bisequence on 3 symbols. Then let $f(i, j)=c_{i-j}$. Thus the horizontal bisequence $\left\{f\left(i, j_{0}\right)\right\}_{i=-\infty}^{\infty}$ is $\left\{c_{i-j_{0}}\right\}_{i=-\infty}^{\infty}$, which is simply a shift of $\mathbf{w}$, and hence non-repetitive. Similarly, the vertical bisequence $\left\{f\left(i_{0}, j\right)\right\}_{j=-\infty}^{\infty}$ is $\left\{c_{i_{0}-j}\right\}_{j=-\infty}^{\infty}$, which is a shifted version of $\mathbf{w}^{R}$, the reverse of $\mathbf{w}$, which again is non-repetitive. (See Figure 1.)

The construction of the proof of Lemma 1.1 labels each horizontal row of lattice points with the non-repetitive bisequence $\mathbf{w}$, and shifts the sequence by one unit as we move vertically from row to row. Notice that the sequences along the positive diagonals are constant! This hardly seems what one wants in a 'non-repetitive tiling'. More appealing would be a tiling in which the sequences on every diagonal were also repetition-free. To

\footnotetext{
${ }^{1}$ An infinite word $a_{1} a_{2} a_{3} \cdots$ can be formalized as an infinite sequence $\left\{a_{i}\right\}_{i=1}^{\infty}$. Similarly, doubly infinite words $\cdots a_{-1} a_{0} a_{1} a_{2} a_{3} \cdots$ correspond to bisequences $\left\{a_{i}\right\}_{i=-\infty}^{\infty}$. A subword of such a bisequence is any finite string $a_{i_{0}} a_{i_{0}+1} \cdots a_{i_{0}+j_{0}}$ where $i_{0} \in \mathbb{Z}, j_{0} \in \mathbb{Z} \geq 0$.
} 


$\begin{array}{ccccccc} & & & & & \vdots \\ & 1 & 2 & 3 & 1 & 3 & 2 \\ & 2 & 3 & 1 & 3 & 2 & 1 \\ & 3 & 1 & 3 & 2 & 1 & 2 \\ & 1 & 3 & 2 & 1 & 2 & 3 \\ & 3 & 2 & 1 & 2 & 3 & 2 \\ & 2 & 1 & 2 & 3 & 2 & 1 \\ & & & & & \vdots\end{array}$

Figure 1: A 'non-repetitive tiling'

use the language of chess, the result of [1] is that with 3 symbols an infinite chessboard can be labelled so that any rook move scans a non-repetitive word. In the present paper we will seek labellings for which any queen move scans a non-repetitive word.

Definition 1.2 A non-repetitive tiling of $\mathbb{Z}^{n}$ by $s$ symbols is a labelling $f: \mathbb{Z}^{n} \rightarrow$ $\{1,2, \ldots, s\}$, for some natural number $s$, such that if $P$ is any $n$-tuple of integers and $Q$ any $n$-tuple whose entries are from the set $\{0,-1,1\}$ but with $Q$ not equal to $(0,0,0, \ldots, 0)$, then the bisequence $\{f(P+i Q)\}_{i=-\infty}^{\infty}$ is non-repetitive.

We will prove the following:

Theorem 1.3 There is a non-repetitive tiling of $\mathbb{Z}^{2}$ using 5 symbols. There is no such tiling with fewer than 5 symbols.

The problem remains open for $n>2$ :

Open Problem 1.4 What is the least $s$ such that there is a non-repetitive tiling of $\mathbb{Z}^{n}$ using $s$ symbols?

Lemma 1.5 Suppose that there is a non-repetitive tiling of $\mathbb{Z}^{2}$ by symbols. Then $s \geq 5$.

Proof: Suppose for the sake of a contradiction that there is such a tiling with $s \leq 4$. Since $\{f(i, 1)\}_{i=-\infty}^{\infty}$ is non-repetitive, there must be at least 3 distinct symbols among the $f(i, 1)$. It follows that for some value of $i$, the symbols $f(i, 1), f(i+1,1), f(i+2,1)$ will be distinct. Without loss of generality we can suppose that $f(0,1)=1, f(1,1)=2$, $f(2,1)=3$. The non-repetitiveness of the tiling requires that adjacent symbols be distinct. This implies that $f(1,0)=f(1,2)=4$. We must then have $f(0,2)=3, f(2,2)=1$. Then $f(1,3)$ is adjacent to 3,4 and 1 , and so must be 2 . But then $f(1,0) f(1,1) f(1,2) f(1,3)=$ 4242 , which is a repetition. This contradiction proves the lemma.

Definition 1.6 Let $\mathbf{w}=\left\{c_{i}\right\}_{i=-\infty}^{\infty}$ be any bisequence. If $k \in \mathbb{N}$, the $\bmod k$ subsequences of $\mathbf{w}$ are those bisequences of the form $\mathbf{w}_{k j}=\left\{c_{i k+j}\right\}_{i=-\infty}^{\infty}, 0 \leq j \leq k-1$. A repetition in a $\bmod k$ subsequence of $\mathbf{w}$ is called a repetition $\bmod k$ of $\mathbf{w}$. We say that $\mathbf{w}$ is non-repetitive up to $\bmod k$ if $\mathbf{w}$ has no repetition $\bmod r$ for any $r, 1 \leq r \leq k$. 
Given a natural number $k$, let $m(k)$ be the least $s$ such that there is a bisequence over $s$ symbols which is non-repetitive up to $\bmod k$. We have the following problem:

Open Problem 1.7 What are the values of $m(k)$ ?

For $k=1$, 'non-repetitive up to $\bmod k$ ' is the same as 'non-repetitive', so that by Thue [6], $m(1)=3$. In this paper we prove that $m(2)=4$ and $m(3)=5$. Definition 1.6 is motivated by generalizing the construction in the proof of Lemma 1.1:

Let $\mathbf{w}=\left\{c_{k}\right\}_{k=-\infty}^{\infty}$ be any bisequence. Consider the labelling of $\mathbb{Z}^{2}$ given by $f(i, j)=$ $c_{i-2 j}$. The horizontal subsequences in this labelling of the plane, $\left\{f\left(i, j_{0}\right)\right\}_{i=-\infty}^{\infty}, j_{0} \in \mathbb{Z}$ look like $\left\{c_{i-2 j_{0}}\right\}_{i=-\infty}^{\infty}$, shifted copies of $\mathbf{w}$. The vertical subsequences $\left\{f\left(i_{0}, j\right)\right\}_{j=-\infty}^{\infty}$, $i_{0} \in \mathbb{Z}$ look like $\left\{c_{i_{0}-2 j}\right\}_{j=-\infty}^{\infty}$, shifted copies of either $\mathbf{w}_{20}^{R}$ or $\mathbf{w}_{21}^{R}$, depending on whether $i_{0}$ is even or odd, respectively. The positive diagonal sequences $\left\{f\left(i, j_{0}+i\right)\right\}_{i=-\infty}^{\infty}, j_{0} \in \mathbb{Z}$ look like $\left\{c_{i-2\left(i+j_{0}\right)}\right\}_{j=-\infty}^{\infty}=\left\{c_{-i-2 j_{0}}\right\}_{i=-\infty}^{\infty}$, shifted versions of $\mathbf{w}^{R}$. The negative diagonal sequences $\left\{f\left(i, j_{0}-i\right)\right\}_{i=-\infty}^{\infty}, j_{0} \in \mathbb{Z}$ look like $\left\{c_{i-2\left(j_{0}-i\right)}\right\}_{j=-\infty}^{\infty}=\left\{c_{3 i-2 j_{0}}\right\}_{i=-\infty}^{\infty}$, shifted copies of $\mathbf{w}_{3 j}, j=0,1,2$.

We thus have the following result:

Lemma 1.8 If there is a bisequence $\mathbf{w}$ on symbols such that $\mathbf{w}$ is non-repetitive up to mod 3, then there is a non-repetitive tiling of $\mathbb{Z}^{2}$ with s symbols.

\section{Other Lattices}

In this paper we are chiefly concerned with the lattice $\mathbb{Z}^{2}$ but in this section we briefly discuss generalizations to other lattices.

Lemma 1.8 can be generalized to $n$ dimensions.

Lemma 2.1 Let $\mathbf{w}=\{c(i)\}_{i=-\infty}^{\infty}$ be an infinite bisequence on symbols which is nonrepetitive up to $\bmod 2^{n}-1$. Let $f: \mathbb{Z}^{n} \rightarrow\{1,2, \ldots, s\}$ be given by $f\left(x_{1}, x_{2}, \ldots, x_{n}\right)=$ $c\left(\sum_{j=1}^{n} 2^{j-1} x_{j}\right)$. Then $f$ gives a non-repetitive tiling of $\mathbb{Z}^{n}$ with s symbols.

Proof: Let $P=\left(p_{1}, p_{2} \ldots, p_{n}\right), Q=\left(q_{1}, q_{2} \ldots, q_{n}\right)$ where $q_{i} \in\{-1,0,1\}$ for each $i$. Let $\mathbf{W}=\{f(P+i Q)\}_{i=-\infty}^{\infty}=\left\{c\left(\sum_{j=1}^{n} 2^{j-1} p_{j}+i \sum_{j=1}^{n} 2^{j-1} q_{j}\right)\right\}_{i=-\infty}^{\infty} . \mathbf{W}$ is thus a shifted version of one of the $\bmod k$ subsequences of $\mathbf{w}$, or of $\mathbf{w}^{R}$, where $k=\left|\sum_{j=1}^{n} 2^{j-1} q_{j}\right|$. However, $0<\left|\sum_{j=1}^{n} 2^{j-1} q_{j}\right| \leq 2^{n}-1$, so that $\mathbf{W}$ must be non-repetitive, since $\mathbf{w}$ is non-repetitive up to mod $2^{n}-1$.

There is an easy lower bound on $s$ in Lemma 2.1; in a non-repetitive labelling of $Z^{n}$, the $2^{n}$ points with all coordinates either 0 or 1 must receive different labels.

Lemma 2.2 Any non-repetitive tiling of $\mathbb{Z}^{n}$ must use at least $2^{n}$ symbols.

The bound in Lemma 2.2 is not sharp in the cases $n=1$ and $n=2$. In these cases we will see that $2^{n}+1$ symbols are needed. We have not been able to show that more than 8 symbols are needed in the three dimensional case. 


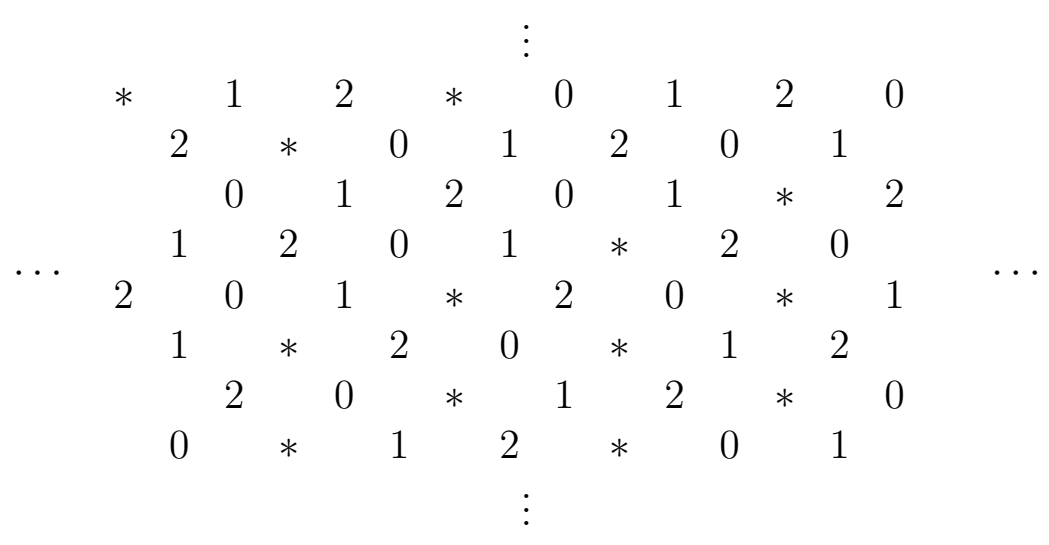

Figure 2: A triangular tiling

One can study lattices other than $\mathbb{Z}^{n}$. For example, label the rows of a triangular lattice with a bisequence $\mathbf{v}=\{c(i)\}_{i=-\infty}^{\infty}$ which is non-repetitive up to mod 2 so that the label $c(1)$ in one row lies above and between labels $c(2)$ and $c(3)$ in the row below. (See Figure 2.) Such a sequence is obtained in Section 4. Then the labels along lines with slope $3^{1 / 2} / 2$ form the word $\mathbf{v}^{R}$, while the labels along lines with slope $-3^{1 / 2} / 2$ form the word $\mathbf{v}_{02}$ or $\mathbf{v}_{12}$. If these are all repetition-free, then the lattice has no repetitions in the three main directions.

\section{A word on 5 symbols which is non-repetitive up to $\bmod 3$.}

To build infinite words which are non-repetitive up to $\bmod k$, we restrict our attention to certain highly structured words.

Definition 3.1 A perturbed $k$-cycle is a word $\mathbf{W}$ over $\{1,2, \ldots, k, *\}$ such that

1. every two-letter subword of $\mathbf{W}$ contains the symbol $*$ at most once

2. if $x y$ is any two letter subword of $\mathbf{W}$ not containing the symbol $*$ then $y \equiv x+1$ $\bmod k$.

3. if $x * z$ is a three letter subword of $\mathbf{W}$, then $z \equiv x+1 \bmod k$.

In a perturbed $k$-cycle the symbols $123 \cdots k 123 \cdots k \cdots$ repeat over and over again, cyclically, with the pattern occasionally broken by the appearance of the symbol $*$. For example, $2 * 31 * 231231 * 231 * 2 * 3 *$ is a perturbed 3 -cycle. An early result of Thue [7] is (with relabelling) that there are infinite non-repetitive words over $\{1,2, *\}$ not having $1^{*} 1$ or $2^{*} 2$ as subwords. Such words are perturbed 2-cycles. 
Lemma 3.2 (Thue) There is an infinite perturbed 2-cycle which is non-repetitive up to $\bmod 1$.

We will also prove the following:

Lemma 3.3 There is an infinite perturbed 3-cycle which is non-repetitive up to mod 2.

Lemma 3.4 There is an infinite perturbed 4-cycle which is non-repetitive up to mod 3.

These results are optimal, in the sense that an infinite perturbed $k$-cycle cannot be non-repetitive up to mod $k$. This suggests the following problem:

Open Problem 3.5 For which $k$ is there a perturbed $(k+1)$-cycle which is non-repetitive up to $\bmod k$ ?

Definition 3.6 Define substitutions $f:\{0,1\} \rightarrow\{0,1\}^{*}$ and $g:\{0,1\} \rightarrow\{a, b\}^{*}$ by

$$
\begin{aligned}
& f(0)=01 \\
& f(1)=00001
\end{aligned} \text { and } \begin{aligned}
g(0) & =a b b b \\
g(1) & =a b b b b b b
\end{aligned}
$$

If $x$ is a letter and $\mathbf{u}$ a word, denote by $|\mathbf{u}|_{x}$ the number of $x$ 's in $\mathbf{u}$.

In what follows let $\mathbf{w}$ be the $\omega$-word $f^{\omega}(0)$. Let $\mathbf{W}$ be the word obtained from $g(\mathbf{w})$ by replacing each $a$ by a ${ }^{*}$, and the $i^{\text {th }}$ occurrence of $b$ in $\mathbf{w}$ by the least positive residue of $i$ modulo 4. Thus

$$
\begin{gathered}
\mathbf{w}=01000010101010100001010000101000010100001010000101010101 \cdots \\
g(\mathbf{w})=a b b b a b b b b b b a b b b a b b b a b b b a b b b a b b b b b b a b b b a b b b b b b a b b b a b b b b b b \cdots
\end{gathered}
$$

and

$$
\mathbf{W}=* 123 * 012301 * 230 * 123 * 012 * 301 * 230123 * 012 * 301201 * 230 * 123012 \ldots
$$

We will show in Theorem 3.17 that $\mathbf{W}$ is non-repetitive up to mod 3. First we obtain some technical results about the word $\mathbf{W}$.

Remark 3.7 Every finite subword of $\mathbf{w}$ appears in $\mathbf{w}$ infinitely often. If $1 \mathbf{v} 1$ appears in $\mathbf{w}$ then there exists a word $\mathbf{u}$ in $\mathbf{w}$ such that $f(\mathbf{u})=\mathbf{v} 1$, if $0000 \mathbf{v} 1$ appears then there exists $\mathbf{u}$ such that $f(\mathbf{u})=0000 \mathbf{v} 1$ and if $1 \mathbf{v} 00001$ appears then there exists $\mathbf{u}$ such that $f(\mathbf{u})=\mathbf{v}$.

Definition 3.8 If $\mathbf{u}$ is a finite subword of $\mathbf{w}$ then $h(\mathbf{u})=\left(|\mathbf{u}|_{0},|\mathbf{u}|_{1}\right)$.

Lemma 3.9 If $\mathbf{u}$ is a finite subword of $\mathbf{w}$ then $h(f(\mathbf{u}))=\left(|\mathbf{u}|_{0}+4|\mathbf{u}|_{1},|\mathbf{u}|_{0}+|\mathbf{u}|_{1}\right)$.

Proof: Clearly the number of occurrences of 0 in $f(\mathbf{u})$ is $|\mathbf{u}|_{0}+4|\mathbf{u}|_{1}$ and the number of occurrences of 1 is $|\mathbf{u}|_{0}+|\mathbf{u}|_{1}$. 
Lemma 3.10 If $\mathbf{w}$ contains a subword $\mathbf{u} 0 \mathbf{u},|\mathbf{u}|_{1} \geq 1$ and

$$
h(\mathbf{u}) \cdot(3,2)+3 \equiv 0 \quad(\bmod 4)
$$

then either $\mathbf{w}$ contains a subword $\mathbf{x} 0 \mathbf{x}$ with

$$
h(\mathbf{x}) \cdot(3,2)+3 \equiv 0 \quad(\bmod 4)
$$

and $|\mathbf{x}|<|\mathbf{u}|$ or it contains a subword $\mathbf{x} 1 \mathbf{x}$ with

$$
h(\mathbf{x}) \cdot(3,2)+2 \equiv 0 \quad(\bmod 4)
$$

and $|\mathbf{x}|<|\mathbf{u}|$.

Proof: Suppose $\mathbf{u} 0 \mathbf{u}$ occurs in $\mathbf{w}$. Then the central 0 is the central element of a subword 101, 001, 100 or 000. We consider these possibilities as Cases I, II, III and IV respectively.

Case I It is clear that the $\mathbf{u} 0 \mathbf{u}$ must have the form $10 \mathbf{v} 1010 \mathbf{v} 1$ for some subword $\mathbf{v}$. By the Remark 3.7 there exists $\mathbf{x}$ in $\mathbf{w}$ such that $f(\mathbf{x})=0 \mathbf{v} 1$. Thus $\mathbf{u} 0 \mathbf{u}=1 f(\mathbf{x}) 01 f(\mathbf{x})=$ $1 f(\mathbf{x}) f(0) f(\mathbf{x})=1 f(\mathbf{x} 0 \mathbf{x})$. Suppose that $h(\mathbf{x})=(i, j)$. Then $h(\mathbf{x} 0 \mathbf{x})=(2 i+1,2 j)$ and by Lemma $3.9, h(f(\mathbf{x} 0 \mathbf{x}))=(2 i+8 j+1,2 i+2 j+1)$ so that

$$
\begin{aligned}
h(\mathbf{u} 0 \mathbf{u}) & =h(1 f(\mathbf{x} 0 \mathbf{x})) \\
& =(2 i+8 j+1,2 i+2 j+2) .
\end{aligned}
$$

Since $h(\mathbf{u} 0 \mathbf{u})=h(\mathbf{u})+h(0)+h(\mathbf{u})$ this implies that $h(\mathbf{u})=(i+4 j, i+j+1)$. Now if $h(\mathbf{u}) \cdot(3,2)+3 \equiv 0 \quad(\bmod 4)$ we have

$$
\begin{aligned}
(i+4 j, i+j+1) \cdot(3,2)+3 \equiv 0 \quad(\bmod 4) \\
\quad \Rightarrow 5 i+14 j+5 \equiv 0 \quad(\bmod 4) \\
\quad \Rightarrow i+2 j+1 \equiv 0 \quad(\bmod 4) \\
\quad \Rightarrow-i-2 j-1 \equiv 0 \quad(\bmod 4) \\
\quad \Rightarrow 3 i+2 j+3 \equiv 0 \quad(\bmod 4) \\
\quad \Rightarrow h(\mathbf{x}) \cdot(3,2)+3 \equiv 0 \quad(\bmod 4) .
\end{aligned}
$$

Since $|\mathbf{x}|<|\mathbf{u}|$, $\mathbf{x}$ satisfies the statement of the Lemma. The analysis in the other cases is similar and for these we will only give the main steps.

Case II In this case there must exist a subword $\mathbf{v}$ in $\mathbf{w}$ such that $\mathbf{u} 0 \mathbf{u}=1 \mathbf{v} 00001 \mathbf{v} 000$, and there then exists $\mathbf{x}$ such that $f(\mathbf{x})=\mathbf{v}$. Thus $\mathbf{u} 0 \mathbf{u}=1 f(\mathbf{x} 1 \mathbf{x}) 000$. If $h(\mathbf{x})=(i, j)$ we get $h(f(\mathbf{x} 1 \mathbf{x}))=(2 i+8 j+4,2 i+2 j+1)$ and $h(\mathbf{u})=(i+4 j+3, i+j+1)$. Then

$$
(i+4 j+3, i+j+1) \cdot(3,2)+3 \equiv 0 \quad(\bmod 4)
$$




$$
\begin{aligned}
& \Rightarrow i+2 j+2 \equiv 0 \quad(\bmod 4) \\
& \Rightarrow 3 i+2 j+2 \equiv 0 \quad(\bmod 4) \\
& \Rightarrow h(\mathbf{x}) \cdot(3,2)+2 \equiv 0 \quad(\bmod 4) .
\end{aligned}
$$

and again the Lemma is satisfied.

Case III In this case there must exist a subword $\mathbf{v}$ in $\mathbf{w}$ such that

$$
\mathbf{u} 0 \mathbf{u}=0001 \mathbf{v} 0100001 \mathbf{v} 01
$$

and then there exists $\mathbf{x}$ such that $f(\mathbf{x})=\mathbf{v} 01$. Thus $\mathbf{u} 0 \mathbf{u}=0001 f(\mathbf{x} 1 \mathbf{x})$. If $h(\mathbf{u}) \cdot(3,2)+3 \equiv$ $0 \quad(\bmod 4)$ we get, as in the last case, that

$$
h(\mathbf{x}) \cdot(3,2)+2 \equiv 0 \quad(\bmod 4)
$$

as required.

Case IV A slight complication occurs here since we do not know whether the subword 000 defining the case is the first or second occurrence of 000 in a subword 00001 We can handle both possibilities at once by saying that $\mathbf{u} 0 \mathbf{u}$ must have the form zvy0zvy where $\mathbf{y z}=0001$ and $\mathbf{z}$ is non-empty. Then $\mathbf{u} 0 \mathbf{u}=\mathbf{z v} 00001 \mathbf{v y}$ and there exists $\mathbf{x}$ in $\mathbf{w}$ such that $\mathbf{u} 0 \mathbf{u}=\mathbf{z} f(\mathbf{x} 1 \mathbf{x}) \mathbf{y}$. Then if $h(\mathbf{x})=(i, j)$ and $h(\mathbf{u}) \cdot(3,2)+3 \equiv 0 \quad(\bmod 4)$ we get

$$
h(f(\mathbf{x} 1 \mathbf{x}))=(2 i+8 j+4,2 i+2 j+1)
$$

and

$$
\begin{aligned}
h(\mathbf{y} f(\mathbf{x} 1 \mathbf{x}) \mathbf{z}) & =h(\mathbf{y})+h(f(\mathbf{x} 1 \mathbf{x}))+h(\mathbf{z}) \\
& =h(\mathbf{y z})+h(f(\mathbf{x} 1 \mathbf{x})) \\
& =(3,1)+(2 i+8 j+4,2 i+2 j+1) \\
& =(2 i+8 j+7,2 i+2 j+2) .
\end{aligned}
$$

As in cases II and III we get $h(\mathbf{x}) \cdot(3,2)+2 \equiv 0 \quad(\bmod 4)$ as required.

Lemma 3.11 If $\mathbf{w}$ contains a subword $\mathbf{u} 1 \mathbf{u},|\mathbf{u}|_{1} \geq 1$ and

$$
h(\mathbf{u}) \cdot(3,2)+2 \equiv 0 \quad(\bmod 4)
$$

then either $\mathbf{w}$ contains a subword $\mathbf{x} 0 \mathbf{x}$ with

$$
h(\mathbf{x}) \cdot(3,2)+3 \equiv 0 \quad(\bmod 4)
$$

and $|\mathbf{x}|<|\mathbf{u}|$ or $\mathbf{w}$ contains a subword $\mathbf{x} 1 \mathbf{x}$ with

$$
h(\mathbf{x}) \cdot(3,2)+3 \equiv 0 \quad(\bmod 4)
$$

and $|\mathbf{x}|<|\mathbf{u}|$. 
Proof: The proof here uses the same ideas as that of the previous Lemma. We consider the three cases in which the central 1 of $\mathbf{u} 1 \mathbf{u}$ is the center of 10101,00101 or 10100. We cannot have 00100 occurring in $\mathbf{w}$ since this would mean we had 0000100001 which could only appear as the image of 11 under $f$ and it is clear that 11 does not occur in $\mathbf{W}$.

Case I In this case $\mathbf{u}$ cannot be 010 or 01010 since this would not satisfy $h(\mathbf{u}) \cdot(3,2)+$ $2 \equiv 0 \quad(\bmod 4)$, so $\mathbf{w}$ must contain $\mathbf{u} 1 \mathbf{u}=010 \mathbf{v} 0101010 \mathbf{v} 010$ which has the form $f(\mathbf{x} 0 \mathbf{x}) 0$. If $h(\mathbf{x})=(i, j)$ then $h((f(\mathbf{x} 0 \mathbf{x}) 0)=(2 i+8 j+2,2 i+2 j+1)$ and $h(\mathbf{u})=i+4 j+1, i+j)$. If $h(\mathbf{u}) \cdot(3,2)+2 \equiv 0 \quad(\bmod 4)$ then $i+2 j+1 \quad(\bmod 4)$. But then, as in the first case of the previous Lemma, $3 i+2 j+3 \equiv 0 \quad(\bmod 4)$, that is, $h(\mathbf{x}) \cdot(3,2)+3 \equiv 0 \quad(\bmod 4)$.

Case II This time $\mathbf{w}$ contains $\mathbf{u} 1 \mathbf{u}=01 \mathbf{v} 0000101 \mathbf{v} 0000$. This must be followed by 1 so $\mathbf{w}$ contains $\mathbf{u} 1 \mathbf{u} 1=01 \mathbf{v} 0000101 \mathbf{v} 00001$ which equals $01 f(\mathbf{x} 0 \mathbf{x})$ for some $\mathbf{x}$. If $h(\mathbf{x})=(i, j)$ then $h(\mathbf{u} 1 \mathbf{u} 1)=(2 i+8 j+2,2 i+2 j+2)$ so that $h(\mathbf{u})=(i+4 j+1, i+j)$. If $h(\mathbf{u}) \cdot(3,2)+2 \equiv 0 \quad(\bmod 4)$ then $h(\mathbf{x}) \cdot(3,2)+2 \equiv i+2 j+1 \quad(\bmod 4)$, which is congruent to 0 modulo 4 as in case I.

Case III Now $\mathbf{w}$ contains $00001 \mathbf{v} 10100001 \mathbf{v} 10=f(\mathbf{x} 0 \mathbf{x}) 0$. The case then follows as in Case I.

Theorem 3.12 Word $\mathbf{w}$ does not contain a subword $\mathbf{u} 0 \mathbf{u}$ with

$$
h(\mathbf{u}) \cdot(3,2)+3 \equiv 0 \quad(\bmod 4)
$$

or a subword $\mathbf{u} 1 \mathbf{u}$ with

$$
h(\mathbf{u}) \cdot(3,2)+2 \equiv 0 \quad(\bmod 4) .
$$

Proof: Suppose that $\mathbf{w}$ does contain such a subword. Consider the case in which $|\mathbf{u}|$ is minimal. By the previous two Lemmas $\mathbf{u}$ cannot contain a 1. The only possibilities for $\mathbf{u} 0 \mathbf{u}$ are then 000 or 0 (with $\mathbf{u}$ empty) and the only possibilities for $\mathbf{u} 1 \mathbf{u}$ are 010 and 1. None of these satisfies the specified congruence.

Corollary 3.13 Word $\mathbf{w}$ does not contain a subword $\mathbf{u} 00 \mathbf{u}$ with $h(\mathbf{u}) \cdot(3,2)+2 \equiv 0$ $(\bmod 4)$

Proof: Suppose otherwise and that $\mathbf{u} 00 \mathbf{u}$ is a counterexample to the Corollary. Neither $\mathbf{u}=0$ nor $\mathbf{u}$ empty is a counterexample so we conclude that $|\mathbf{u}|_{1} \geq 1$. Then the 00 must be contained in 00001 in $\mathbf{u} 00 \mathbf{u}$. Thus we may write $\mathbf{u} 00 \mathbf{u}$ as $\mathbf{d x c} 00 \mathbf{d x c}$ where $\mathbf{c d}=001$ and $\mathbf{d}$ is non-empty. There then exists $\mathbf{z}$ such that $\mathbf{x}=f(\mathbf{z})$, and

$$
\begin{aligned}
\mathbf{u} 00 \mathbf{u} & =\mathbf{d} f(\mathbf{z}) 0001 f(\mathbf{z}) \mathbf{c} \\
& =\mathbf{d} f(\mathbf{z} 1 \mathbf{z}) \mathbf{c} .
\end{aligned}
$$

Suppose that $h(\mathbf{z})=(i, j)$. Then $h(\mathbf{z} 1 \mathbf{z})=(2 i, 2 j+1), h(f(\mathbf{z} 1 \mathbf{z})=(2 i+8 j+4,2 i+2 j+1)$, and

$$
\begin{aligned}
h(\mathbf{u} 00 \mathbf{u}) & =h(\mathbf{d} f(\mathbf{z} 1 \mathbf{z}) \mathbf{c}) \\
& =h(f(\mathbf{z} 1 \mathbf{z})+h(\mathbf{c d}) \\
& =(2 i+8 j+4,2 i+2 j+1)+(2,1) \\
& =(2 i+8 j+6,2 i+2 j+2) .
\end{aligned}
$$


We conclude that $h(\mathbf{u})=(i+4 j+2, i+j+1)$. If $h(\mathbf{u}) \cdot(3,2)+2 \equiv 0 \quad(\bmod 4)$ we get $i+2 j+2 \equiv 0(\bmod 4)$ which implies that $3 i+2 j+2 \equiv 0(\bmod 4)$, that is $h(\mathbf{z}) \cdot(3,2)+2 \equiv 0 \quad(\bmod 4)$ which contradicts the Theorem.

We now show that the word $\mathbf{W}$ contains no repetitions up to mod 3 .

Lemma $3.14 \mathrm{~W}$ contains no mod 1 repetition.

Proof: Suppose $\mathbf{W}$ contains repetitions and let $\mathbf{v v}$ be the first. Then there is a corresponding repetition $\mathbf{y} c \mathbf{y} c$ in the word $g(\mathbf{w})$ where $c$ is a letter and $\mathbf{y}$ is a (possibly empty) subword. We must have $|\mathbf{y} c|_{b} \equiv 0 \quad(\bmod 4)$, for if not, the first $b$ in the first $\mathbf{y}$ will not be mapped onto the same digit as the first $b$ in the second $\mathbf{y}$. We cannot have $c$ preceding $\mathbf{y} c \mathbf{y} c$ in $g(\mathbf{w})$ for then $c \mathbf{y} c \mathbf{y}$ would occur in $g(\mathbf{w})$ and this would be mapped into a repetition in $\mathbf{W}$ which occurs earlier than $\mathbf{v v}$. It is not hard to see that $\mathbf{y} c \mathbf{y} c$ then has the form (a) bbbzabbbza or (b) bbbzabbbbbbzabbb where $\mathbf{z}$ is a subword and $\mathbf{z}=g(\mathbf{u})$ for some subword $\mathbf{u}$ of $\mathbf{w}$. Thus $\mathbf{y} c \mathbf{y} c$ has the form (a) $b b b g(\mathbf{u} 0 \mathbf{u}) a$ or (b) $b b b g(\mathbf{u} 1 \mathbf{u}) a b b b$.

In case (a) we have $|\mathbf{z}|_{b}+3 \equiv 0 \quad(\bmod 4)$. Recalling the mapping $g$ we see that we must then have

$$
3|\mathbf{u}|_{0}+6|\mathbf{u}|_{1}+3 \equiv 0 \quad(\bmod 4)
$$

so that $\mathbf{w}$ contains a subword $\mathbf{u} 0 \mathbf{u}$ with $3|\mathbf{u}|_{0}+2|\mathbf{u}|_{1}+3 \equiv 0 \quad(\bmod 4)$. This is impossible by Theorem 3.12 .

In case (b) we have $|\mathbf{z}|_{b}+6 \equiv 0 \quad(\bmod 4)$ and there exists $\mathbf{u} 1 \mathbf{u}$ in $g(\mathbf{w})$ with

$$
3|\mathbf{u}|_{0}+6|\mathbf{u}|_{1}+6 \equiv 0 \quad(\bmod 4) .
$$

This is equivalent to $3|\mathbf{u}|_{0}+2|\mathbf{u}|_{1}+2 \equiv 0 \quad(\bmod 4)$ which is also prohibited by Theorem 3.12 .

Lemma 3.15 W contains no mod 2 repetition.

Proof: Suppose $\mathbf{W}$ contains a subword $c_{1} d_{1} c_{2} \ldots d_{2 n-1} c_{2 n}$ where $c_{i}=c_{i+n}$ for $i=$ $1, \ldots, n$, so that $c[1 . .2 n]$ is a mod 2 repetition. We note that the values of $c_{i}$ and $c_{i+1}$ uniquely determine the value of $d_{i}$, for instance if $c_{i}=0$ and $c_{i+1}=*$ then $d_{i}=1$. We thus have $d_{i}=d_{i+1}$ for $i=1, \ldots, n-1$ and the whole subword has the form $\mathbf{v} d_{n} \mathbf{v}$. The pre-image of this in $g(\mathbf{w})$ has the form $\mathbf{y} c \mathbf{y}$ where $c$ is a letter and $\mathbf{y}$ is a subword. As in the previous Lemma we have $|\mathbf{y} c|_{b} \equiv 0 \quad(\bmod 4)$. We cannot have $\mathbf{y} c \mathbf{y}$ preceded by $c$ as this would produce a mod 1 repetition in $\mathbf{W}$. This means that $\mathbf{y} c \mathbf{y}$ has the form $b b b \mathbf{z} a b b b a b b b \mathbf{z} a b b b$ where $\mathbf{z}=g(\mathbf{u})$ for some subword $\mathbf{u}$ of $\mathbf{w}$. Word $\mathbf{w}$ thus contains a subword $\mathbf{u} 00 \mathbf{u}$ where

$$
\begin{aligned}
0 & \equiv|\mathbf{y} c|_{b} \\
& \equiv|g(0 \mathbf{u} 0)|_{b} \\
& \equiv h(0 \mathbf{u} 0) \cdot(3,2) \\
& \equiv[h(\mathbf{u})+h(00)] \cdot(3,2) \\
& \equiv h(\mathbf{u}) \cdot(3,2)+2 \quad(\bmod 4)
\end{aligned}
$$

which is impossible by Corollary 3.13. 


\section{Lemma $3.16 \mathrm{~W}$ contains no mod 3 repetition.}

Proof: Suppose $\mathbf{W}$ contains a subword $c_{1} d_{1} e_{1} c_{2} \ldots d_{2 n-1} e_{2 n-1} c_{2 n}$ where $c_{i}=c_{i+n}$ for $i=1, \ldots, n$, so that $c[1 . .2 n]$ is a $\bmod 3$ repetition. We assume that $n \geq 2$ since we can see by inspection that $\mathbf{W}$ contains no mod 3 repetitions of the form $c_{1} d_{1} e_{1} c_{1}$. In this case the values of $c_{i}$ and $c_{i+1}$ do not in general uniquely determine the value of $d_{i}$ and $e_{i}$. However they do if either of $c_{i}$ and $c_{i+1}$ is a $*$ or if $c_{i+1}-c_{i} \equiv 3(\bmod 4)$. We also note that the values of $c_{i}, d_{i}, e_{i}, c_{i+1}$ and $c_{i+2}$ determine the values of $d_{i+1}$ and $e_{i+1}$. Ambiguity only arises when $c_{i+1}-c_{i} \equiv 2(\bmod 4)$ for then either $d_{i}$ or $e_{i}$ is a $*$ but we cannot tell which.

This ambiguity disappears with knowledge of four consecutive $c$ values for if $c_{i+1}-c_{i} \equiv$ $2(\bmod 4)$ and $c_{i+2}-c_{i+1} \equiv 2(\bmod 4)$ then we must have $d_{i}=*$ and $e_{i+1}=*$ since the non-* letters come in blocks of length 3 or 6 . The rest of the subword $c_{i} d_{i} \ldots c_{i+3}$ is then determined. If $c_{i+1}-c_{i} \equiv 2(\bmod 4), c_{i+2}-c_{i+1} \equiv 2 \quad(\bmod 4)$ and $c_{i+3}-c_{i+2} \equiv 2$ $(\bmod 4)$ then we must have $d_{i}=*$ and $e_{i+2}=*$ and again the rest of the subword is determined. There are several other cases to consider and the reader can readily check that in these too the subword is completely determined.

We can thus assume that $d_{i}=d_{i+n}$ and $c_{i}=c_{i+n}$ for $i=1 . . n-1$ and the whole subword has the form $\mathbf{v} d_{n} e_{n} \mathbf{v}$. The preimage of this in $g(\mathbf{w})$ has the form $\mathbf{x} d e \mathbf{x}$. As in Lemma 3.14 we have

$$
|\mathbf{x} d e|_{b} \equiv 0 \quad(\bmod 4)
$$

The letter preceding $\mathbf{x} d e \mathbf{x}$ in cannot be $e$ for then we'd have a subword $e \mathbf{x} d e \mathbf{x}$ and obtain a contradiction as in Lemma 3.15. We consider two cases.

Case I Suppose the word $g(\mathbf{w})$ contains a subword of the form $a \mathbf{x} d b \mathbf{x}$. Now $a$ is always followed by $b^{3} a$ or $b^{6} a$ so $\mathbf{x}$ begins with one of these. It cannot begin with the second for then the subword would contain $b^{7}$ which is impossible. Thus $\mathbf{x}$ has the form $b^{3} a \mathbf{y}$ and our subword is $a b^{3} a \mathbf{y} d b^{4} a \mathbf{y}$. It is now clear that $d=b$ and $\mathbf{y}$ has suffix $a b$, so the subword is $a b^{3} a \mathbf{z} a b^{6} a \mathbf{z} a b$. This must then be followed by $b^{2}$ so $g(\mathbf{w})$ contains the repetition $\left(b^{3} a \mathbf{z} a b^{3}\right)^{2}$. Noting that $|\mathbf{z}|_{b}=|\mathbf{y}|_{b}-1=|\mathbf{x}|_{b}-4$ and recalling that $|\mathbf{x} d e|_{b}=\left|\mathbf{x} b^{2}\right|_{b} \equiv 0$ $(\bmod 4)$ we have $\left|b^{3} a \mathbf{z} a b^{3}\right|_{b} \equiv 0 \quad(\bmod 4)$. Thus $\left(b^{3} a \mathbf{z} a b^{3}\right)^{2}$ is the preimage of a mod 1 repetition in $\mathbf{W}$, which is impossible by Lemma 3.14 .

Case II Suppose the word $g(\mathbf{w})$ contains a subword of the form $b \mathbf{x} d a \mathbf{x}$. Using reasoning similar to that used in Case I we see that we must have $d=b$ and $\mathbf{x}$ has prefix $b^{3} a$ and suffix $b^{2}$, say $\mathbf{x}=b^{3} a \mathbf{y} b^{2}$. Thus the subword has the form $b\left(b^{3} a \mathbf{y} b^{2}\right) b a\left(b^{3} a \mathbf{y} b^{2}\right)$. If this is followed by $b a$ we get $\left(b^{3} a \mathbf{y} b^{3} a\right)^{2}$ in $g(\mathbf{w})$ and since $\left|b^{3} a \mathbf{y} b^{3} a\right|_{b}=|\mathbf{y}|_{b}+6=$ $|\mathbf{x}|_{b}-5+6 \equiv 0 \quad(\bmod 4)$ we'd have a mod 1 repetition in $\mathbf{W}$ which is impossible. We conclude that it is followed by $b^{4}$ and $\mathbf{y}$ has suffix $a$ Say $\mathbf{y}=\mathbf{z} a$. We now have the subword $b^{4} a \mathbf{z}\left(a b^{3}\right)\left(a b^{3}\right) a \mathbf{z} a b^{6}$. Then $a \mathbf{z}$ is the image of some subword $\mathbf{u}$ in $\mathbf{w}$ and $a b^{3}=g(0)$ so $g(\mathbf{w})$ contains $g(\mathbf{u} 00 \mathbf{u})$. As before we find that $|a \mathbf{z}|_{b}=|\mathbf{y}|_{b}=|\mathbf{x}|_{b}-5 \equiv 2 \quad(\bmod 4)$. Thus $\mathbf{w}$ contains $\mathbf{u} 00 \mathbf{u}$ with $3|\mathbf{u}|_{0}+6|\mathbf{u}|_{1} \equiv 2 \quad(\bmod 4)$ and thus $h(\mathbf{u}) \cdot(3,2) \equiv 2 \quad(\bmod 4)$. But this is impossible by Corollary 3.13.

We conclude that $\mathbf{W}$ contains no mod 3 repetition. $\square$

Thus we obtain our main result.

Theorem 3.17 W is non-repetitive up to mod 3. 
Proof: Immediate from the last three Lemmas. $\square$

We use this to prove Theorem 1.3.

Proof of Theorem 1.3: We must show that a non-repetitive tiling of $\mathbb{Z}^{2}$ exists using 5 symbols, but not with less than 5 . We showed in Lemma 1.5 that at least 5 symbols are required. That 5 are sufficient follows from Lemma 1.8 and Theorem 3.17.

\section{A word on 4 symbols which is non-repetitive up to $\bmod 2$.}

Definition 4.1 Define substitution $h:\{0,1\} \rightarrow\{a, b\}^{*}$ by

$$
\begin{aligned}
& h(0)=a b b \\
& h(1)=a b b b b b
\end{aligned}
$$

We begin with the word $\mathbf{w}$ defined in the last section. Let $\mathbf{V}$ be the word obtained from $h(\mathbf{w})$ by replacing each $a$ by a ${ }^{*}$, and the $i^{t h}$ occurrence of $b$ in $\mathbf{w}$ by the least residue of $i$ modulo 3. Thus

$$
\begin{gathered}
\mathbf{w}=010000101010101000010100001010000101000010100 \cdots \\
h(\mathbf{w})=\text { abbabbbbbabbabbabbabbabbbbbabbabbbbbabbabbbbb } \cdots
\end{gathered}
$$

and

$$
\mathbf{V}=* 12 * 01201 * 20 * 12 * 01 * 20 * 12012 * 01 * 20120 * 12 * 01201 \cdots
$$

Using the methods of the previous section one also shows that the word $\mathbf{V}$ is nonrepetitive up to mod 2. It is easy to check, as in the proof of Lemma 1.5, that no such word exists on 3 symbols. Thus we have proved:

Theorem 4.2 The least $s$ such that there is a bisequence over s symbols which is nonrepetitive up to $\bmod 2$ is 4 .

\section{References}

[1] Dwight R. Bean, Andrzej Ehrenfeucht and George McNulty, Avoidable Patterns in Strings of Symbols, Pacific J. Math. 85 (1979), 261-294.

[2] Stanley Burris and Evelyn Nelson, Embedding the dual of $\Pi_{\infty}$ in the lattice of equational classes of semigroups, Algebra Universalis, 1 (1971/72), 248-253; MR 45 \#5257.

[3] Andres del Junco, A transformation with simple spectrum which is not rank one, Canad. J. Math. 29 (1977), 655-663; MR 57 \#6367.

[4] Marston Morse and Gustav A. Hedlund, Symbolic dynamics I, II, Amer. J. Math. 60 (1938), 815-866; 62 (1940) 142; MR 1, 123d. 
[5] P. S. Novikov and S. I. Adjan, Infinite periodic groups I, II, III, Izv. Akad. Nauk. SSSR Ser. Mat. 32 (1968), 212-244;251-524;709-731;MR 39 \#1532a-c.

[6] Axel Thue, Über unendliche Zeichenreihen, Norske Vid. Selsk. Skr. I. Mat. Nat. Kl. Christiana (1906), 1-22.

[7] Axel Thue, Über unendliche Zeichenreihen, Norske Vid. Selsk. Skr. I. Mat. Nat. Kl. Christiana (1912), 1-67. 